\title{
Early Clinical Outcomes of a New Posteriorly Stabilized Total Knee Arthroplasty Prosthesis: Comparisons with Two Established Prostheses
}

\author{
Nimesh P. Jain, $\mathrm{MS}^{1}$, Sung Yup Lee, $\mathrm{MD}^{2}$, Vivek M. Morey, $\mathrm{MS}^{3}$, Suri Chong, $\mathrm{MD}^{2}$, Yeon Gwi Kang, $\mathrm{MS}^{2}$, and \\ Tae Kyun Kim, MD ${ }^{2}$ \\ ${ }^{1}$ Department Orthopaedics, North Cumbria University Hospitals NHS Trust, Carlisle, UK; ${ }^{2}$ Department of Orthopaedic Surgery, Seoul National University Bundang \\ Hospital, Seongnam, Korea; ${ }^{3}$ Department of Orthopedics, Datta Meghe Institute of Medical Sciences, Wardha, India
}

\begin{abstract}
Purpose: We sought to determine whether early clinical performance of new posterior stabilized (PS) knee system, the Vega-PS (Aesculap), is better than that of two established total knee arthroplasty (TKA) prostheses, the E.motion-PS (Aesculap) and the Genesis II (Smith \& Nephew) in terms of functional outcomes, patient satisfaction, and incidence of adverse events.

Materials and Methods: We compared the clinical outcomes of 206 consecutive TKAs using Vega-PS with those of 205 TKAs using E.motion-PS and 216 TKAs using Genesis II at 2 years of follow-up.

Results: Overall, the knees with the Vega-PS had better functional outcome scores than the knees with the E.motion-PS, but had similar outcome scores to the knees with the Genesis II, as evident from the American Knee Society knee score (94.2 vs. 92.5 vs. 93.2), Western Ontario McMaster Universities Osteoarthritis (WOMAC) stiffness index (1.8 vs. 2.3 vs. 2.0), WOMAC function index (11.8 vs. 16.8 vs. 18.5), Short Form 36 (SF-36) physical component summary score ( 41.9 vs. 39.3 vs. 41.6 ), and SF-36 mental component summary score (50.0 vs. 45.8 vs. 46.9 ). Patient satisfaction was higher in the Vega-PS and Genesis II groups than the E.motion-PS group. No notable group differences were found in terms of the incidence of adverse events.

Conclusions: The Vega-PS, a newly developed PS fixed bearing prosthesis, had comparable or superior clinical performance in comparison with the two established fixed or mobile bearing PS prostheses.
\end{abstract}

Keywords: Arthroplasty, Replacement, Knee, Prosthesis design, Outcome assessment

\section{Introduction}

Total knee arthroplasty (TKA) has emerged as a durable approach to replicate the healthy osseous anatomy and restore natural knee joint kinematics. However, recent expansion of indi-

Received September 1, 2016; Revised (1st) January 6, 2017;

(2nd) March 12, 2017; Accepted April 7, 2017

Correspondence to: Tae Kyun Kim, MD

Department of Orthopaedic Surgery, Joint Reconstruction Center,

Seoul National University Bundang Hospital, 82 Gumi-ro 173 beon-gil,

Bundang-gu, Seongnam 13620, Korea

Tel: +82-31-787-7196, Fax: +82-31-787-4056

E-mail: osktk@snubh.org

This is an Open Access article distributed under the terms of the Creative Commons Attribution Non-Commercial License (http://creativecommons.org/licenses/by-nc/4.0/) which permits unrestricted non-commercial use, distribution, and reproduction in any medium, provided the original work is properly cited. cations for TKA in a younger and more active patient population demands further improvement for greater durability ${ }^{1,2)}$ and function $^{3,4)}$. Many new implant designs have incorporated modifications to achieve the goal of better clinical outcomes and implant longevity. However, these designs do not guarantee improved results, and several new prostheses were reported to have unexpected problems or unsatisfactory outcomes ${ }^{5-7)}$. Therefore, with any new design, it is prudent to maintain a close surveillance of its performance and safety from the outset. Although a shortterm outcome analysis is limited in predicting long-term success, information from short-term studies is still valuable for early detection of adverse events, if any, originating from new prostheses. Posterior stabilized (PS) knee prostheses have cam and post mechanisms to provide stability in flexion, increase range of motion (ROM), and improve the quadriceps lever arm. Moreover, multiple mid-term and long-term follow-up studies have evi- 
denced good function, movement, and survivorship of PS knee prostheses ${ }^{8,9)}$. However, certain drawbacks have been reported with PS knee prostheses, namely large intercondylar bone stock removal to accommodate the femoral $\mathrm{cam}^{10}$, dislocation of the $\mathrm{cam}^{11)}$, patellar clunk syndrome ${ }^{12)}$, and wear of the tibial post ${ }^{13)}$. Subsequently, there have been many attempts to modify the designs of PS knee prostheses in order to address their problems as well as to achieve more physiologic and stable knee kinematics. Several studies in the past have shown that changes in component geometry and modularity with the PS designs have led to improved short- and long-term results ${ }^{14-16)}$ in addition to permitting greater surgical flexibility in severe osteoarthritis cases ${ }^{16)}$.

A new PS knee prosthesis system, Vega-PS (Aesculap; B. Braun, Tuttlingen, Germany), is designed to improve TKA outcomes. Its distinguishing characteristics include a low profile intercondylar box, reduced posterior condyle length, and narrowed mediolateral width of the femoral component, along with an anterior cutout and increased post inclination of the tibial insert. It was designed to achieve high performance, including high flexion and shape optimization to avoid overhang. However, no clinical information regarding this new prosthesis is available yet to validate its functional performance and safety. We sought to determine whether early clinical performance of this new prosthesis is better than two established TKA prostheses, the E.motion-PS and Genesis II, in terms of functional outcomes, patient satisfaction, and incidence of adverse events. We had two hypotheses: 1) The functional outcomes and patient satisfaction in patients having Vega-PS prostheses implanted are better than the outcomes and satisfaction in patients having the previously established PS type TKA prostheses implanted. 2) There is no increased incidence of adverse events in the knees replaced with the Vega-PS.

\section{Materials and Methods}

Seven hundred and eighty-six TKAs were performed by one surgeon (KTK) between May 2006 and May 2012. These included 240 TKAs using a new PS knee system, the Vega-PS (Fig. 1), along with 546 consecutive TKAs performed for advanced osteoarthritis using two established PS knee systems, the E.motionPS (Aesculap), a mobile bearing PS prosthesis (257 knees), and the Genesis II (Smith \& Nephew, Memphis, TN, USA), a fixed bearing PS prosthesis (289 knees). All of the Vega-PS TKAs were performed in the years after we completed the 257 E.motion-PS TKAs. In contrast, the 289 Genesis II TKAs were done throughout the entire study period. The implant selection was at the surgeon's discretion without any selection criteria. We included only patients with a diagnosis of primary osteoarthritis, scheduled for unilateral TKA, between May 2006 and May 2012. We excluded patients with a diagnosis of secondary osteoarthritis or a past medical history of previous hip or spine surgery, in an attempt to reduce potential outcome confounders. A total of 159 patients, including 16 patients with a history of prior hip and spine problems, 24 patients with secondary arthritis, 89 patients with other systemic comorbidities that might interfere with the benefits of the replaced knee, 15 patients with postoperative complications affecting outcomes, and 15 patients who died due to unrelated causes, were excluded from the study. Thus, data from a total of 627 patients were used for analysis (Vega-PS, n=206; E.motion-PS, $\mathrm{n}=205$; and Genesis II, $\mathrm{n}=216$ ). In the Vega-PS group, the mean age of the patients was 70.7 years and the number of female patients was 198 (96.1\%). In the E.motion-PS group, the mean age of the patients was 68.3 years, and the number of female patients was 196 (95.6\%), while in the Genesis II group, the mean age was
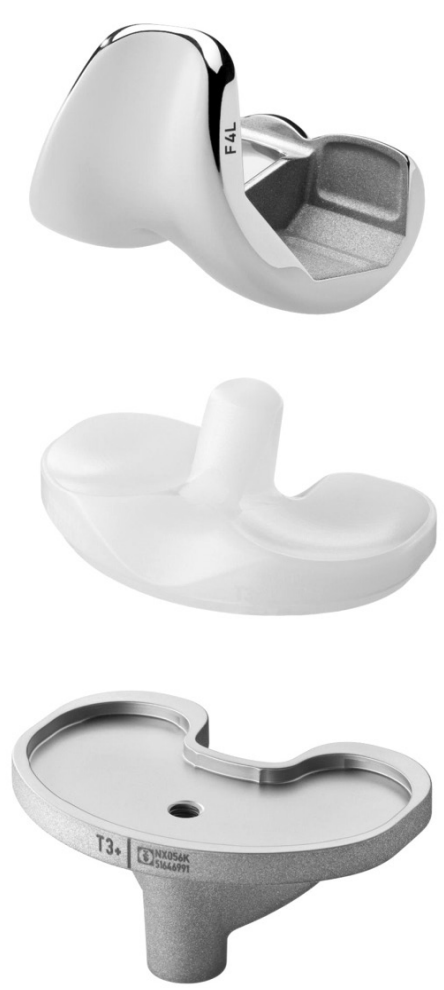

Fig. 1. The photographs show the Vega-PS prosthesis. The anterior and posterior narrowing of the femoral component with a low profile intercondylar box minimizes interference with the surrounding soft tissues. Fifty-five degrees posterior inclination of the post acts to avoid postedge loading for better load distribution, prevents impingement with the extensor mechanism, and increases resistance to dislocation. The deepened anterior cutout of the insert allows smooth articulation with the patellar tendon. 
69.7 years, and the number of female patients was 203 (98.5\%). This study was approved by the Institutional Review Board of our hospital, and all patients provided informed consent concerning the use of medical records.

All surgeries were performed by a single surgeon, using the medial parapatellar approach. The patella was routinely resurfaced and all implants were fixed with cement (Palacos; Heraeus Kulzer $\mathrm{GmbH}$, Hanau, Germany). We used measured resection, combined with gap balancing techniques without navigation.

There were not any significant differences in surgical techniques between the mobile and fixed bearing instruments. After surgery, a compressive dressing was applied, with immobilization of the knee for 24 hours. The knees were then placed in a continuous passive-motion machine. On the second postoperative day, the drain and compressive dressing were removed, and all patients were encouraged to walk with crutches or a walker and started active and passive ROM exercises. The knee ROM exercises and weight bearing were gradually increased.

All clinical information was prospectively collected, using predesigned data sheets, and maintained in a database by an independent investigator (LSY). The retrospective evaluation of all the data was done with a minimum follow-up of 2 years. The clinical information gathered included demographic data, preoperative clinical status, and postoperative clinical status at 12 months and 2 years. Patient evaluation using the outcome scales was done at 2 years after surgery. For this purpose, knee ROM, American Knee Society (AKS) scores ${ }^{17}$, Western Ontario McMaster Universities Osteoarthritis Index (WOMAC) scales ${ }^{18)}$, and Short Form 36 (SF-36) scores $^{19)}$ were used. In addition, patient satisfaction was evaluated at the 2 year follow-up using a 1-4 point Likert scale, based on the grading system developed by the British Orthopaedic Association, which is divided into four levels, i.e., enthusiastic, satisfied, not committed, and disappointed ${ }^{20)}$. The knee motion arc was expressed in terms of flexion contracture and maximum flexion angle. An independent investigator (LSY) measured the flexion contracture and maximum flexion angles to the nearest $5^{\circ}$ by using a standard $(38 \mathrm{~cm})$ clinical goniometer, with the patient in the supine position.

The radiographic assessment was performed at 2 years after surgery to measure five parameters: mechanical tibiofemoral angle (MTFA), coronal femoral prosthesis alignment, coronal tibial prosthesis alignment, sagittal femoral prosthesis alignment, and sagittal tibial prosthesis alignment (Fig. 2). Two of the authors took all radiographic measurements using full-length weight bearing anteroposterior radiographs, and lateral radiographs of the operated limb. To achieve the same foot rotation
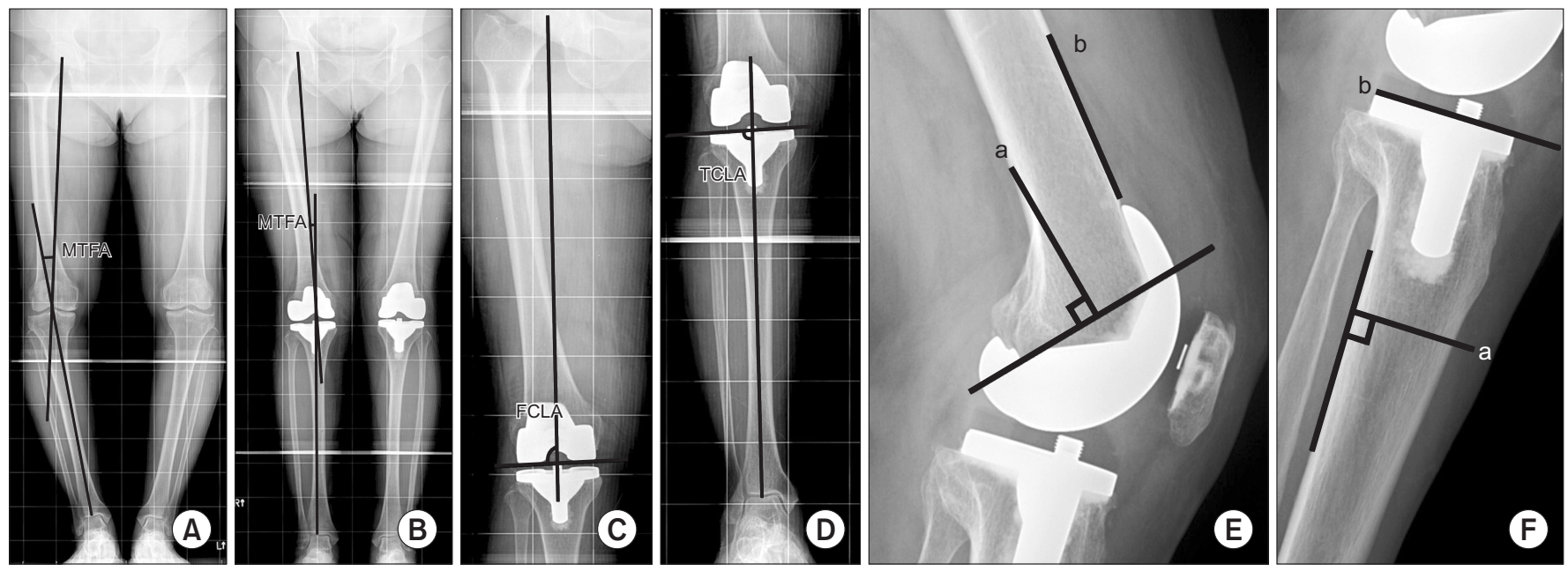

Fig. 2. (A) The radiograph shows the preoperative mechanical tibiofemoral angle (MTFA). (B) The radiograph shows the postoperative MTFA. (C) The radiograph shows the femoral component lateral angle (FCLA), defined as the lateral angle between the femoral mechanical axis and a line connecting the most distal points of the medial and lateral condyles of the femoral component. (D) The radiograph shows the the tibial component lateral angle (TCLA), defined as the lateral angle between the tibial mechanical axis and a line parallel to the top surface of the tibial component. (E) The radiograph shows the femoral component flexion angle. To assess the sagittal alignment of the femoral component, we measured the angle between the line perpendicular to the tangent line of the femoral component box (a) and the line connecting the anterior cortical point of $10 \mathrm{~cm}$ proximal to the joint line and the anterior point of the meta-epiphysis junction before metaphyseal diverging (b). (F) The radiograph shows the tibial component posterior slope. To assess the sagittal alignment of the tibial component, we measured the angle between the line parallel to the upper surface of the tibial tray (b) and the line perpendicular to the tangent line of the anatomical axis (the posterior cortical line of tibia at two points, $5 \mathrm{~cm}$ and $15 \mathrm{~cm}$ distal to the joint line) of the proximal tibia (a). 
angle, a reference template was positioned on the platform of our plain radiographic system. All radiographic images were digitally acquired using a picture archiving and communication system (PACS; Infinitt, Seoul, Korea). Assessments were performed on a 20-inch LCD monitor in portrait mode using the PACS software. To determine intra- and inter-observer reliabilities of the radiographic assessments, the two investigators performed all radiographic assessments in 20 randomly selected radiographs twice, with an interval of 1 week. The intra- and inter-observer reliabilities were then evaluated using intraclass correlation coefficients, which were found to be $>0.80$ for all measurements. Since the measurements were judged as reliable, measurements taken by a single investigator (LSY) were used in the final analyses.

In order to gauge overall limb alignment, we measured the MTFA of the knee, which was defined as the angle formed by the intersection between the mechanical axis of the femur (the line from the femoral head center to the femoral intercondylar notch center) and the tibia (the line from the ankle talus center to the center of tibial spine tips) (Fig. 2A and B). A negative value was given to the angle of the knees in varus alignment. To assess the coronal alignment of the femoral component, we measured the femoral component lateral angle (FCLA), defined as the lateral angle between the femoral mechanical axis and a line connecting the most distal points of the medial and lateral condyles of the femoral component (Fig. 2C). The coronal femoral prosthesis alignment was calculated by subtracting the FCLA from 90, and accordingly, a negative value was given to the angle of varus orientation of the femoral component. To assess the coronal alignment of the tibial component, we measured the tibial component lateral angle, (TCLA) defined as the lateral angle between the tibial mechanical axis and the line parallel to the top surface of the tibial component (Fig. 2D). We calculated the coronal tibial prosthesis alignment by subtracting the TCLA from $90^{\circ}$, and accordingly, a negative value was assigned to the angle for varus orientation of the tibial component. To assess the sagittal alignment of the femoral component, we measured the angle between

Table 1. Comparison of Demographic Characteristics, Preoperative Mechanical Axis (MA) and Outcome Scales among the Vega-PS, E.motion-PS, and Genesis II Groups

\begin{tabular}{|c|c|c|c|c|c|c|c|}
\hline \multirow{2}{*}{ Variable } & \multirow{2}{*}{$\begin{array}{l}\text { Vega-PS } \\
(n=206)\end{array}$} & \multirow{2}{*}{$\begin{array}{l}\text { E.motion-PS } \\
\qquad(\mathrm{n}=205)\end{array}$} & \multirow{2}{*}{$\begin{array}{c}\text { Genesis II } \\
(n=216)\end{array}$} & \multirow{2}{*}{$\mathrm{p}$-value } & \multicolumn{3}{|c|}{ p-value $e^{a)}$} \\
\hline & & & & & $\mathrm{P}-\mathrm{V}$ & V-G & P-G \\
\hline Sex (female, \%) & $198(96.1)$ & $196(95.6)$ & $203(98.5)$ & 0.199 & & & \\
\hline Height (cm) & $151.5(6.0)$ & $151.9(6.0)$ & $151.5(5.8)$ & 0.733 & & & \\
\hline Weight (kg) & $61.5(8.7)$ & $63.0(8.2)$ & $63.9(9.9)$ & 0.023 & $>0.05$ & 0.020 & $>0.05$ \\
\hline Preoperative MA $\left(^{\circ}\right)$ & $-10.7(4.8)$ & $-10.1(5.1)$ & $-11.6(6.7)$ & 0.827 & & & \\
\hline \multicolumn{8}{|l|}{ Motion $\operatorname{arc}\left({ }^{\circ}\right)$} \\
\hline Flexion contracture & $11.2(6.3)$ & $9.9(6.5)$ & $12.0(6.6)$ & $0.006(0.022)$ & $0.152(0.223)$ & $0.005(0.019)$ & $0.685(1.000)$ \\
\hline Maximum flexion & $135.6(13.8)$ & $135.9(13.5)$ & $135.0(14.2)$ & $0.527(0.782)$ & & & \\
\hline \multicolumn{8}{|l|}{ WOMAC } \\
\hline Pain & $9.8(4.3)$ & $12.2(4.6)$ & $11.0(4.5)$ & $<0.001(<0.001)$ & $<0.001(<0.001)$ & $0.032(0.036)$ & $0.016(0.017)$ \\
\hline Stiffness & $4.4(2.1)$ & $4.9(2.0)$ & $4.4(1.9)$ & $0.011(0.013)$ & $0.031(0.050)$ & $0.021(0.019)$ & $1.000(1.000)$ \\
\hline Function & $34.1(13.9)$ & $41.2(14.5)$ & $39.6(12.2)$ & $<0.001(<0.001)$ & $<0.001(<0.001)$ & $0.813(0.667)$ & $<0.001(<0.001)$ \\
\hline \multicolumn{8}{|l|}{ Short Form 36} \\
\hline PCS & $31.6(8.0)$ & $29.8(7.1)$ & $30.1(8.1)$ & $0.051(0.053)$ & & & \\
\hline MCS & $48.4(11.5)$ & $41.1(12.3)$ & $41.6(12.3)$ & $<0.001(<0.001)$ & $<0.001(<0.001)$ & $1.000(1.000)$ & $<0.001(<0.001)$ \\
\hline
\end{tabular}

Values are presented as mean (standard deviation).

P-V: E.motion-PS vs. Vega-PS, P-G: E.motion-PS vs. Genesis II, V-G: Vega-PS vs. Genesis II, AKS: American Knee Society, WOMAC: Western Ontario McMaster Universities Osteoarthritis Index, PCS: physical component summary, MCS: mental component summary. a) $\mathrm{p}$-value is listed along with the adjusted $\mathrm{p}$-value (after taking the preoperative differences as covariates). 
the line perpendicular to the tangent line of the femoral component box and the line connecting the anterior cortical point of 10 $\mathrm{cm}$ proximal to the joint line and the anterior point of the metaepiphysis junction before metaphyseal diverging (Fig. 2E). A negative value was given to the angle for extension of the femoral component, while a positive value was given to the angle for flexion of the component. To assess the sagittal alignment of the tibial component, we measured the angle between the line parallel to the upper surface of the tibial tray and the line perpendicular to the tangent line of the anatomical axis (the posterior cortical line of the tibia at two points, $5 \mathrm{~cm}$ and $15 \mathrm{~cm}$ distal to the joint line) of the proximal tibia (Fig. 2F). A positive value was given to the posterior slope of the tibial component and a negative value was given to the anterior slope of the component.

Statistical analyses were carried out using SPSS ver. 21.0 (IBM Co., Armonk, NY, USA), and a p-value $<0.05$ was considered significant. Knees with the Vega-PS were compared to knees with the E.motion-PS and knees with the Genesis II for functional outcome scales and incidence of adverse events. Patient satisfaction was also measured and compared. The Kolmogorov-Smirnov test was used to confirm that the clinical outcome scores, including maximal flexion, were normally distributed. As preoperative differences could have confounding effects on postoperative functional outcomes, all three groups were compared with respect to their demographic characteristics and preoperative clinical status (Table 1). There were a few parameters, which were different among the groups, and their confounding effects were adjusted using the analysis of covariance (ANCOVA) test, when comparing postoperative outcomes. To determine the significance of the differences in the functional outcome scores among the three implant systems, post hoc analysis was performed.

To determine adequacy of our sample size, we performed an a priori power analysis using the two-sided hypothesis test at an alpha level of 0.05 . Sixty-four knees were required to detect a difference of $5^{\circ}$ in a motion arc and a $6 \%$ difference in outcome scales. We considered these cutoff values to be clinically important because motion arc was measured to the nearest $5^{\circ}$, and a $6 \%$ difference of maximum score has been suggested as the minimal clinically important difference for WOMAC and SF-36 indices ${ }^{14)}$. Thus, the sample sizes used were regarded as adequate.

\section{Results}

Knees replaced with the Vega-PS had comparable functional outcomes with the Genesis II but better results than the E.motion-PS. In the knees with the Vega-PS, almost all of the measured outcome scales improved at 2 years postoperatively $(p<0.05)$ (Table 2). On comparisons among the three groups, the mean AKS knee score (Vega-PS, 94.2; E.motion-PS, 92.5; and Genesis II, 93.2) ( $\mathrm{p}=0.046)$ and WOMAC stiffness score (VegaPS, 1.8; E.motion-PS, 2.3; and Genesis II, 2.0) $(\mathrm{p}=0.020)$ were found to be better for the knees replaced with the Vega-PS than for the knees with the E.motion-PS (Table 3). Although knees replaced with the Vega-PS and Genesis II revealed similar results in terms of most of the outcome scores, the Vega-PS knees had better WOMAC function scores compared with the Genesis II group (Vega-PS, 11.8; E.motion-PS, 16.8; and Genesis II, 18.5) $(\mathrm{p}<0.001)$. Conversely, knees replaced with the Genesis II had better AKS function scores than knees with the Vega-PS and knees with the E.motion-PS (Genesis II, 95.7; Vega-PS, 93.0; E.motion-PS, 93.6) ( $\mathrm{p}=0.022)$. Nevertheless, the three groups showed similar results in terms of flexion contracture, maximal flexion achieved, WOMAC pain score, SF-36 mental component summary (MCS) scores, and physical component summary (PCS) scores (Table 3). Patient satisfaction was higher in the Vega-PS and Genesis II groups than the E.motion-PS group ( $\mathrm{p}=0.001$ ) (Table 4). No notable differences were found in limb and prosthesis alignment among the three groups postoperatively (Table 5).

Table 2. Comparison of Preoperative and 2-Year Postoperative Outcomes of the Vega-PS Prosthesis

\begin{tabular}{lccc}
\hline \multicolumn{1}{c}{ Variable } & Preoperative & $\begin{array}{c}\text { Postoperative } \\
\text { 2-year }\end{array}$ & p-value \\
\hline Motion arc $\left(^{\circ}\right)$ & & & \\
Flexion contracture & $11.2(6.3)$ & $0.3(1.5)$ & $<0.001$ \\
Maximum flexion & $135.6(13.8)$ & $132.4(10.4)$ & $<0.001$ \\
Range of motion & $124.4(17.3)$ & $132.1(10.6)$ & $<0.001$ \\
AKS score & & & \\
Knee & $46.6(9.5)$ & $94.2(4.5)$ & $<0.001$ \\
Function & $58.4(9.8)$ & $93.0(9.2)$ & $<0.001$ \\
WOMAC & & & $<0.001$ \\
Pain & $9.8(4.3)$ & $2.3(2.7)$ & $<0.001$ \\
Stiffness & $4.4(2.1)$ & $1.8(1.5)$ & $<0.001$ \\
Function & $34.1(13.9)$ & $11.8(9.7)$ & \\
Short Form 36 & & & $<0.001$ \\
PCS & $31.6(8.0)$ & $41.9(8.0)$ & \\
MCS & $48.4(11.5)$ & $50.0(11.6)$ & $<0.001$ \\
\hline
\end{tabular}

Values are presented as mean (standard deviation).

AKS: American Knee Society, WOMAC: Western Ontario McMaster Universities Osteoarthritis Index, PCS: physical component summary, MCS: mental component summary. 
Table 3. Comparison of Functional Outcomes among Three Implant Groups at 2 Years after Surgery

\begin{tabular}{|c|c|c|c|c|c|c|c|}
\hline \multirow{2}{*}{ Variable } & \multirow{2}{*}{$\begin{array}{l}\text { Vega-PS } \\
(n=206)\end{array}$} & \multirow{2}{*}{$\begin{array}{l}\text { E.motion-PS } \\
\qquad(n=205)\end{array}$} & \multirow{2}{*}{$\begin{array}{c}\text { Genesis II } \\
(\mathrm{n}=216)\end{array}$} & \multirow{2}{*}{$\mathrm{p}$-value } & \multicolumn{3}{|c|}{ p-value ${ }^{a)}$} \\
\hline & & & & & $\mathrm{P}-\mathrm{V}$ & V-G & P-G \\
\hline \multicolumn{8}{|l|}{ Motion $\operatorname{arc}\left({ }^{\circ}\right)$} \\
\hline Maximum flexion & $132.4(10.4)$ & $130.3(11.1)$ & $130(13.8)$ & $0.155(0.067)$ & & & \\
\hline \multicolumn{8}{|l|}{ AKS score } \\
\hline Function & $93.0(9.2)$ & $93.6(9.1)$ & $95.7(8.2)$ & $0.022(0.003)$ & $1.000(1.000)$ & $0.129(0.011)$ & $0.025(0.007)$ \\
\hline \multicolumn{8}{|l|}{ WOMAC } \\
\hline Pain & $2.3(2.7)$ & $3.0(2.9)$ & $3.0(3.2)$ & $0.034(0.075)$ & $0.077(0.168)$ & $1.000(1.000)$ & $0.082(0.153)$ \\
\hline Stiffness & $1.8(1.5)$ & $2.3(1.5)$ & $2.0(1.6)$ & $0.020(0.004)$ & $0.016(0.003)$ & $0.446(0.449)$ & $0.695(0.238)$ \\
\hline MCS & $50.0(11.6)$ & $45.8(10.3)$ & $46.9(10.6)$ & $0.007(0.065)$ & $0.007(0.063)$ & $1.000(1.000)$ & $0.108(0.509)$ \\
\hline
\end{tabular}

Values are presented as mean (standard deviation).

P-V: E.motion-PS vs. Vega-PS, P-G: E.motion-PS vs. Genesis II, V-G: Vega-PS vs. Genesis II, AKS: American Knee Society, WOMAC: Western Ontario McMaster Universities Osteoarthritis index, PCS: physical component summary, MCS: mental component summary. ${ }^{a} \mathrm{p}$-value is listed along with the adjusted p-value (after taking the preoperative differences as covariates).

Table 4. Comparison of Patient Satisfaction among the Three Implant Groups at 2 Years after Surgery

\begin{tabular}{lcccc}
\hline \multicolumn{1}{c}{ Variable } & $\begin{array}{c}\text { Vega-PS } \\
(\mathrm{n}=206)\end{array}$ & $\begin{array}{c}\text { E.motion-PS } \\
(\mathrm{n}=205)\end{array}$ & $\begin{array}{c}\text { Genesis II } \\
(\mathrm{n}=216)\end{array}$ & p-value \\
\hline Satisfaction score & & & & \\
Enthusiastic & $49(23.8)$ & $18(8.8)$ & $46(21.2)$ & \\
Satisfied & $157(76.2)$ & $173(84.4)$ & $158(73.3)$ & 0.001 \\
Noncommittal & 0 & $14(6.8)$ & $12(5.5)$ & \\
Disappointed & 0 & 0 & 0 & \\
\hline
\end{tabular}

Values are presented as number (\%).

No noticeable differences were noted in the incidence of adverse events: Vega-PS, 5 (2.4\%; immediate postoperative infection 1 , wound complication 2, and periprosthetic fracture 2); E.motionPS, 8 (3.9\%; immediate postoperative infection 1, wound complication 4 , periprosthetic fracture 2, and instability 1); and Genesis II, 2 (0.92\%; immediate postoperative infection 1 and periprosthetic fracture 1) $(\mathrm{p}>0.05)$. All the postoperative infection cases were treated with open debridement with prosthesis retention. Wound complications were treated with prolonged antibiotic administration. All of the periprosthetic fracture cases, which were minimally displaced, were treated conservatively. Instability complications were treated by applying a knee brace for 6 weeks. In addition, radiographic evaluation showed no radiolucent lines
Table 5. Comparison of Limb and Prosthesis Component Alignment between the Three Implant Groups at 2 Years after Surgery

\begin{tabular}{ccccc}
\hline Variable & $\begin{array}{c}\text { Vega-PS } \\
(\mathrm{n}=206)\end{array}$ & $\begin{array}{c}\text { E.motion- } \\
\text { PS }(\mathrm{n}=205)\end{array}$ & $\begin{array}{c}\text { Genesis II } \\
(\mathrm{n}=216)\end{array}$ & p-value \\
\hline $\begin{array}{c}\text { Postoperative } \\
\text { mechanical axis }\left(^{\circ}\right)\end{array}$ & $-0.6(2.4)$ & $-0.6(2.6)$ & $0.2(2.2)$ & 0.110 \\
$\begin{array}{c}\text { Femoral coronal } \\
\text { alignment }\left(^{\circ}\right)\end{array}$ & $-0.7(1.6)$ & $-0.8(1.6)$ & $-0.1(1.7)$ & 0.070 \\
$\begin{array}{c}\text { Tibial coronal } \\
\text { alignment }\left({ }^{\circ}\right)\end{array}$ & $0.3(1.4)$ & $0.4(1.57)$ & $0.6(1.5)$ & 0.850 \\
$\begin{array}{c}\text { Femoral sagittal } \\
\text { alignment }\left(^{\circ}\right)\end{array}$ & $-0.4(2.4)$ & $1.4(2.4)$ & $0.8(2.52)$ & $<0.001$ \\
$\begin{array}{c}\text { Tibial sagittal } \\
\text { alignment }\left({ }^{\circ}\right)\end{array}$ & $1.4(1.3)$ & $0.7(1.5)$ & $2.0(1.9)$ & $<0.001$ \\
\hline
\end{tabular}

Values are presented as mean (standard deviation).

or osteolysis in any of the cases in all three implant groups.

\section{Discussion}

The main finding of this study is that the Vega-PS, a newly developed PS fixed bearing prosthesis, had comparable or superior clinical performances in comparison with the two established fixed or mobile bearing PS prosthesis, without any added incidence of adverse events. PS knee design was developed with 
the intent of achieving better stability in flexion and increased $\mathrm{ROM}^{21}$. Over the past two decades, various implants with enhanced designs of PS knee system prostheses have been introduced, in hopes of improving clinical and functional outcomes and patient satisfaction. The Vega-PS prosthesis was designed recently by Aesculap to achieve high flexion and shape optimization, to avoid overhang. However, whether or not these modifications translate into better clinical performance is unknown. The present study was conducted to compare this new PS knee design with two well-established PS knee systems, the E.motion-PS and the Genesis II, with respect to clinical performance in terms of early functional outcomes, patient satisfaction, and adverse events.

Several limitations of the study should be noted when interpreting our findings. First, our patient population is predominantly female, which could be a confounding factor when our findings are extrapolated to other study populations with a different sex composition. However, female sex dominance does reflect the true sex proportions of patients undergoing TKA in Korea ${ }^{22)}$. Second, this study does not address long-term outcomes, and therefore, we were unable to investigate longevity-related issues, such as wear and loosening, which are of real practical importance considering the design features of the three different prostheses. Lack of randomization can be considered as the third limitation. Although all surgeries were performed by the same surgeon and all clinical data were collected by the same clinical investigator, using predesigned data collection sheets, some confounding factors arising from the study design, particularly, the different periods involved, may have affected the study results. However, we attempted to adjust for possible confounders arising from preoperative differences by conducting analysis of covariance. The fourth limitation is that we did not estimate the overhang value directly; therefore, it is inaccurate to estimate the specific result of the shape optimization for a lesser overhang design. However, we assume that the effect is reflected in the clinical outcomes. Nonetheless, our study had several strengths. It was conducted at a tertiary care center specializing in TKA surgeries and included a statistically adequate number of patients in each group, enhancing the generalizability of our results. All the surgeries were performed by a single high volume surgeon, and all the patients were treated according to the standard updated surgical and perioperative protocols of our center. Furthermore, comparison of the new prosthesis with the well-established fixed bearing and mobile bearing TKA designs helps to ensure more precise and valid comparisons. In addition, as the Vega-PS and E.motion-PS prostheses are manufactured by the same company, any manufacturing related issues were addressed properly in the present study.

The study results partially support our first hypothesis in that they revealed reasonably good functional outcomes for the Vega-PS knees, which were indistinguishable from the Genesis II knees, and better than the E.motion-PS knees. In spite of the observed differences in the functional outcomes, there was no difference among the three groups concerning maximal flexion achieved 2 years after surgery. This lack of correlation between maximal flexion and functional outcomes is consistent with the results of previous studies, which demonstrated only a weak correlation between the postoperative maximum flexion and the clinical parameters for pain relief, function, and quality of life ${ }^{4,23)}$. Additionally, it is asserted that a mobile bearing PS prosthesis provides greater maximum flexion, which is attributed to the femoral rollback and the rotation at the interface between the tibial tray and the bottom surface of the inser ${ }^{8,9)}$.

Furthermore, these differences could be explained by the preoperative differences among the three groups. In particular, patients' weight and body mass index (BMI) were significantly lower in the Vega-PS group than in the Genesis II group. In addition, patients in the Vega-PS group had significantly better WOMAC pain, stiffness, and function scores, and SF 36 MCS scores in the preoperative period. Although we attempted to adjust for their confounding effects using analysis of covariance, it might have helped us to achieve only "satisfactory control" over their confounding effects rather than ensuring their complete neutralization. The controversy regarding the superiority of one over the other between fixed bearing and mobile bearing knees may exist in the present study. The realization of the intended design features of the Vega-PS also seemed reflected in patient satisfaction. This finding is consistent with the results of previous studies ${ }^{20,24,25)}$, which have proved that postoperative patient satisfaction is an important predictor of successful outcomes of TKA.

In this study, the Vega-PS had a better clinical performance than the E.motion-PS. The Vega-PS was designed to achieve high flexion and avoid overhang. Its specific features are a low profile intercondylar box, reduced posterior condyle length, and narrowed mediolateral width of the femoral component, along with an anterior cutout and increased post inclination of the tibial insert. These characteristics seem to be reflected in the superior results of the knees with the Vega-PS in the mean AKS knee score and WOMAC stiffness score than the knees with the E.motionPS. Although the knees replaced with the Vega-PS and Genesis II revealed similar results in the outcome scores, the Vega-PS knees had better WOMAC function scores compared the Genesis II 
group. Although not statistically significant, the clinical outcomes of the Vega-PS were better than the Genesis II in most subscales, and patient satisfaction with the Vega-PS was also better than that of the Genesis-PS. The use of high flexion knee prostheses has become more prevalent recently. And it is controversial whether or not the high flexion TKA implants show improved $\mathrm{ROM}^{26)}$. In this study, there was no difference in ROM among the three prostheses. However we suppose that such a design shape contributed indirectly to the functional outcome. Additional studies are required.

Our study also supports our second hypothesis in that knees replaced with the Vega-PS did not have any added incidence of adverse events. No notable differences were seen among the three implant groups with regard to adverse events. An overall average incidence of adverse events of $2.4 \%$, noted in the present study, is consistent with that of the several studies in the past ${ }^{27-29)}$, which have found good early clinical outcomes with few adverse complications, when using newly developed prostheses. However, some studies have reported unsatisfactory and adverse results in short-term analyses ${ }^{5-7,23,30)}$. For example, early results using high flex legacy PS knee prostheses found a high incidence of loosening of the femoral component ${ }^{6,7}$. In our study, examination of serial radiographs at the 2-year follow-up, showed no radiolucent lines, osteolysis, or other sign of loosening in any of the implanted knees. In that context, the Vega-PS was found to be secure in the early postoperative period.

\section{Conclusions}

The present study demonstrates that the Vega-PS prosthesis had improved functional outcomes at the 2-year follow-up, and no notably high incidence of adverse events was observed. Knees implanted with the Vega-PS seemed to have better functional outcomes and higher patient satisfaction than knees with the E.motion-PS, but were comparable to the knees implanted with the Genesis II. Nevertheless, long-term follow-up evaluation is warranted to confirm the performance of this new prosthesis with regard to its overall function and endurance.

\section{Conflict of Interest}

No potential conflict of interest relevant to this article was reported.

\section{References}

1. Buechel FF Sr. Long-term followup after mobile-bearing total knee replacement. Clin Orthop Relat Res. 2002;(404):4050.

2. Dixon MC, Brown RR, Parsch D, Scott RD. Modular fixedbearing total knee arthroplasty with retention of the posterior cruciate ligament: a study of patients followed for a minimum of fifteen years. J Bone Joint Surg Am. 2005;87:598603.

3. Hoffmann C, Gosheger G, Gebert C, Jurgens H, Winkelmann W. Functional results and quality of life after treatment of pelvic sarcomas involving the acetabulum. J Bone Joint Surg Am. 2006;88:575-82.

4. Nicholls MA, Selby JB, Hartford JM. Athletic activity after total joint replacement. Orthopedics. 2002;25:1283-7.

5. Barrack RL, Nakamura SJ, Hopkins SG, Rosenzweig S. Winner of the 2003 James A: Rand Young Investigator's Award: early failure of cementless mobile-bearing total knee arthroplasty. J Arthroplasty. 2004;19(7 Suppl 2):101-6.

6. Cho SD, Youm YS, Park KB. Three- to six-year follow-up results after high-flexion total knee arthroplasty: can we allow passive deep knee bending? Knee Surg Sports Traumatol Arthrosc. 2011;19:899-903.

7. Han HS, Kang SB, Yoon KS. High incidence of loosening of the femoral component in legacy posterior stabilised-flex total knee replacement. J Bone Joint Surg Br. 2007;89:1457-61.

8. Ranawat CS, Luessenhop CP, Rodriguez JA. The press-fit condylar modular total knee system. Four-to-six-year results with a posterior-cruciate-substituting design. J Bone Joint Surg Am. 1997;79:342-8.

9. Stern SH, Insall JN. Posterior stabilized prosthesis: results after follow-up of nine to twelve years. J Bone Joint Surg Am. 1992;74:980-6.

10. Vince KG. Principles of condylar knee arthroplasty: issues evolving. Instr Course Lect. 1993;42:315-24.

11. Kocmond JH, Delp SL, Stern SH. Stability and range of motion of Insall-Burstein condylar prostheses: a computer simulation study. J Arthroplasty. 1995;10:383-8.

12. Hozack WJ, Rothman RH, Booth RE Jr, Balderston RA. The patellar clunk syndrome: a complication of posterior stabilized total knee arthroplasty. Clin Orthop Relat Res. 1989; (241):203-8.

13. Haas BD. Tibial post impingement in posterior-stabilized total knee arthroplasty. Orthopedics. 2006;29(9 Suppl):S83-5.

14. Lachiewicz PF, Soileau ES. The rates of osteolysis and loos- 
ening associated with a modular posterior stabilized knee replacement: results at five to fourteen years. J Bone Joint Surg Am. 2004;86:525-30.

15. Tayot O, Ait Si Selmi T, Neyret P. Results at 11.5 years of a series of 376 posterior stabilized HLS1 total knee replacements: survivorship analysis, and risk factors for failure. Knee. 2001;8:195-205.

16. Thadani PJ, Vince KG, Ortaaslan SG, Blackburn DC, Cudiamat CV. Ten- to 12-year followup of the Insall-Burstein I total knee prosthesis. Clin Orthop Relat Res. 2000;(380):1729.

17. Insall JN, Dorr LD, Scott RD, Scott WN. Rationale of the Knee Society clinical rating system. Clin Orthop Relat Res. 1989;(248):13-4.

18. Bellamy N, Buchanan WW, Goldsmith CH, Campbell J, Stitt LW. Validation study of WOMAC: a health status instrument for measuring clinically important patient relevant outcomes to antirheumatic drug therapy in patients with osteoarthritis of the hip or knee. J Rheumatol. 1988;15:1833-40.

19. Ware JE Jr, Sherbourne CD. The MOS 36-item short-form health survey (SF-36): I. conceptual framework and item selection. Med Care. 1992;30:473-83.

20. Becker R, Döring C, Denecke A, Brosz M. Expectation, satisfaction and clinical outcome of patients after total knee arthroplasty. Knee Surg Sports Traumatol Arthrosc. 2011; 19:1433-41.

21. Insall JN, Lachiewicz PF, Burstein AH. The posterior stabilized condylar prosthesis: a modification of the total condylar design: two to four-year clinical experience. J Bone Joint Surg Am. 1982;64:1317-23.

22. Kim HA, Kim S, Seo YI, Choi HJ, Seong SC, Song YW,
Hunter D, Zhang Y. The epidemiology of total knee replacement in South Korea: national registry data. Rheumatology (Oxford). 2008;47:88-91.

23. Morrison TA, Nyce JD, Macaulay WB, Geller JA. Early adverse results with bicompartmental knee arthroplasty: a prospective cohort comparison to total knee arthroplasty. J Arthroplasty. 2011;26(6 Suppl):35-9.

24. Du H, Tang H, Gu JM, Zhou YX. Patient satisfaction after posterior-stabilized total knee arthroplasty: a functional specific analysis. Knee. 2014;21:866-70.

25. Kwon SK, Kang YG, Kim SJ, Chang CB, Seong SC, Kim TK. Correlations between commonly used clinical outcome scales and patient satisfaction after total knee arthroplasty. J Arthroplasty. 2010;25:1125-30.

26. Murphy M, Journeaux S, Russell T. High-flexion total knee arthroplasty: a systematic review. Int Orthop. 2009;33:88793.

27. Kim TH, Lee DH, Bin SI. The NexGen LPS-flex to the knee prosthesis at a minimum of three years. J Bone Joint Surg Br. 2008;90:1304-10.

28. Kim TK, Cho HJ, Kang YG, Kim SJ, Chang CB. Improved early clinical outcomes of RP/PS mobile-bearing total knee arthroplasties. Clin Orthop Relat Res. 2009;467:2901-10.

29. Long WJ, Levi GS, Scuderi GR. Highly cross-linked polyethylene in posterior stabilized total knee arthroplasty: early results. Orthop Clin North Am. 2012;43:e35-8.

30. Kim YH, Yoon SH, Kim JS. Early outcome of TKA with a medial pivot fixed-bearing prosthesis is worse than with a PFC mobile-bearing prosthesis. Clin Orthop Relat Res. 2009;467:493-503. 